KIDNEY CANCER

\title{
Programmed death ligand 1 regulation in ccRCC
}

The first evidence that von Hippel-Lindau (VHL) mutations positively correlate with programmed death ligand 1 (PD-L1) expression in clear cell renal cell carcinoma (ccRCC) has been presented in European Urology.

Tumours from 32 patients with ccRCC were assessed for VHL mutation status at both alleles, including somatic point mutations, loss of heterozygosity $(\mathrm{LOH})$ and hypermethylation of the $\mathrm{VHL}$ promoter, and then classified according to type of $\mathrm{VHL}$ alteration. Loss of function (LOF) analysis was then conducted, which was based on the type of mutation present and the presence of $\mathrm{LOH}$ or promoter hypermethylation. Using these criteria, $71.9 \%$ of tumours had $\mathrm{LOH}, 65.6 \%$ had two VHL altered alleles and $43.8 \%$ had LOF. Quantitative real-time PCR was then used to assess PDL1 (also known as $C D 274$ ) expression, which was found to be significantly associated with $\mathrm{VHL}$ status regardless of classification.
In vivo analysis of six different $\mathrm{VHL}$ mutants transfected into the VHL-defective 786 -O cell line (which overexpresses hypoxia-inducible factor $2 \alpha$ (HIF- $2 \alpha$ (also known as endothelial PAS domain-containing protein 1)) showed that gradual truncated $\mathrm{VHL}$ protein ( $\mathrm{pVHL}$ ) LOF induces gradual dysregulation of HIF- $2 \alpha$ expression, which positively correlates with PD-L1 messenger RNA (mRNA) and protein expression. Knock down of HIF2A in pVHL-deficient $786-O$ and $A 498$ cells using short interfering RNA significantly decreased PD-L1 mRNA and protein expression.

Investigation of the mechanism by which HIF- $2 \alpha$ regulates PD-L1 expression revealed putative hypoxia response elements (HREs) in human PDL1, with specific binding of HIF- $2 \alpha$ to HRE- 4 on the PDL1 promoter in VHL-deficient, but not in VHL-corrected, 786-O cells, and HRE-4 was shown to be transcriptionally active.
HIF-1 $\alpha$ was also observed to regulate PD-L1 in VHL-mutated RCC4 cells - knock down of HIF-1 and/or HIF-2 induced significant decreases in PD-L1 protein and mRNA expression.

These results show that PD-L1 is a novel target of HIF-2 $\alpha$ and provide evidence of a link between VHL mutations, the HIF-2 $\alpha$ signalling pathway and PD-L1 expression, indicating that the VHL-HIF-2 $\alpha$ axis has a crucial role in the PD-L1 response in this disease.

Louise Stone

ORIGINAL ARTICLE Messai, Y. et al. Renal cell carcinoma programmed death-ligand 1 , a new direct target of hypoxiainducible factor- 2 alpha, is regulated by von Hippel-Lindau gene mutation status. Eur. Urol. http://dx.doi.org/10.1016/ j.eururo.2015.11.029

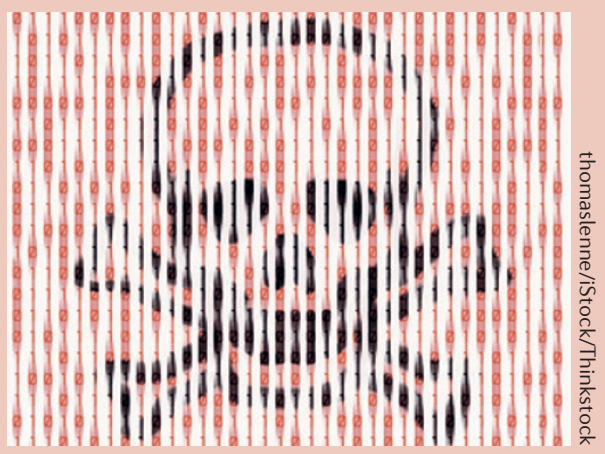

